\title{
APLICAÇÃO DA TÉCNICA DE MANUTENÇÃO CENTRADA EM CONFIABILIDADE, COMO METODOLOGIA NA MANUFATURA SUBSTRATIVA DE USINAGEM EM CILINDROS
}

\author{
L. A. Campos ${ }^{1 *} ;$ R. A. Moura ${ }^{1}$ \\ 1 Faculdade de Tecnologia de São José dos Campos - Professor Jessen Vidal \\ Av. Cesare Mansueto Giulio Lattes, 1350 - Eugênio de Melo, São José dos Campos/SP, \\ CEP.: 12247-014, Brasil. \\ Telefone: (12) 3905-2423 \\ luciana.apcampos@hotmail.com
}

\begin{abstract}
RESUMO: A falta de confiabilidade em máquinas e equipamentos, nas atividades produtivas, causam falhas que comprometem a disponibilidade do equipamento, reduzindo assim a competitividade de tal forma que afetem os negócios da organização. Este trabalho propõe a utilização da ferramenta módulo de falhas e seus efeitos aplicando na manutenção centrada na confiabilidade, respeitando o histórico do equipamento, suas peculiaridades e condições de uso. O método, além de vasta pesquisa literária, contará com a análise, levantamento e histórico necessários para adoção de uma disponibilidade confiável na aplicação de análise de falhas integrado à um torno computadorizado visando reduzir as ocorrências das falhas. $\mathrm{O}$ resultado além, do conhecimento disponível, contribuirá com uma abordagem mais abrangente possível e detalhada, embasada em uma vasta revisão bibliográfica detalhada que culminará com análise crítica. Nesse viés conclui-se que utilizando a metodologia de pesquisa-ação, reduziu as falhas.
\end{abstract}

PALAVRAS-CHAVE: falhas; manutenção centrada; confiabilidade.

\begin{abstract}
The lack of reliability in machinery and equipment, in productive activities, cause failures that compromise the equipment availability, thus reducing the competitiveness in such a way that affect the organization's business. This work proposes the use of the tool failure module and its effects applying in maintenance focused on reliability, respecting the history of the equipment, its peculiarities and conditions of use. The method, in addition to extensive literary research, will include analysis, Survey and history needed to adopt a reliable availability in the application of fault analysis integrated to a computer lathe to reduce the occurrence of failures. The result, in addition to the available knowledge, will contribute to a more comprehensive and detailed approach, based on a comprehensive detailed bibliographic review that will culminate with critical analysis. In this bias it is concluded that using the methodology of action research, reduced the failures.
\end{abstract}

KEYWORDS: Failure Analysis; Centered Maintenance; FMEA; Reliability.

\section{INTRODUÇÃO}

A manutenção centrada em confiabilidade (MCC) ou em inglês reliability centered maintenance (RCM) é o processo utilizado para a determinação da metodologia de manutenção mais efetiva para o tratamento de falhas potenciais e também um método para planejamento de manutenção desenvolvido dentro da indústria aeronáutica e, posteriormente, adaptado a várias outras indústrias e 


\section{ISSN $2447-5378$}

ramos militares. Este artigo apresenta uma abordagem estruturada para o MCC e discute as várias etapas da abordagem. O método RCM fornece uma estrutura para utilizar a experiência operacional de maneira mais sistemática. Os requisitos para modelos de confiabilidade e dados são, portanto, destacados. A lacuna entre os profissionais de manutenção e os cientistas que trabalham com modelos de otimização de manutenção é discutida, juntamente com alguns desafios futuros para a RCM.

Desta forma o desenvolvimento da metodologia MCC em um torno de usinagem para cilindro de laminação envolve a identificação e análise de falhas das quebras durante o processo de usinagem que causaram grandes interrupções, bem como a tomada de ações que quando executadas irão reduzir a indisponibilidade do equipamento, bem como seu custo de manutenção.

Destacamos neste trabalho o diagnóstico do atual modelo de gestão da manutenção, analisando a necessidade de implementar uma nova filosofia baseada na Manutenção Centrada na Confiabilidade. Também como estudo de caso, realizar uma análise e diagnosticar de maneira rápida e eficiente, as falhas potenciais antes que elas aconteçam e determinar as estratégias de manutenção a fim de preservar as funções do equipamento, propiciando aumento da qualidade, disponibilidade, confiabilidade e segurança do sistema, além da diminuição dos custos de manutenção.

\subsection{Equipamento utilizado no estudo de caso}

O equipamento em estudo se trata de um torno horizontal CNC DH 1450 da WALDRICH SIGUEN que estava operando sobre regime de vinte quatro horas com objetivo de cumprir a programação de produção, mas, em virtude da falha no programa de manutenção o equipamento apresentou uma elevação no nível de vibração e sem motivo aparente irregularidades no acabamento final da peça. $\mathrm{O}$ equipamento estudado é apresentado na Figura 1.

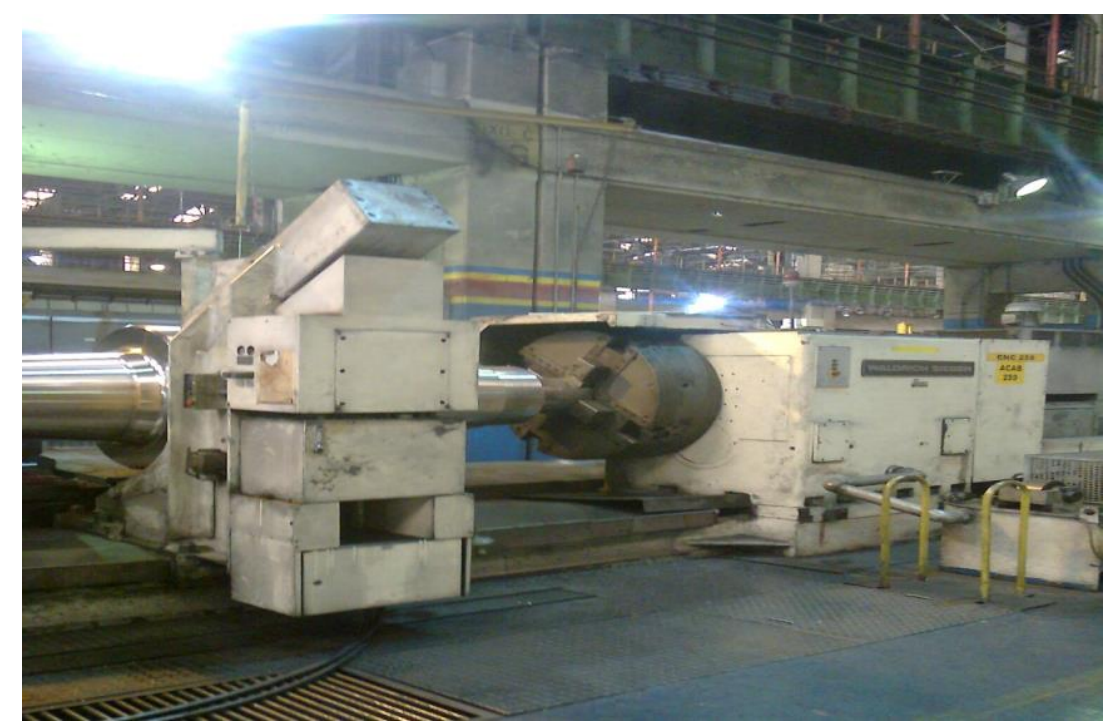

Figura 1. Torno CNC Waldrich Siguen DH 1450

\section{REVISÃO BIBLIOGRÁFICA}

O diferencial de Manutenção Centrada na Confiabilidade é tratar a confiabilidade do ponto de vista quantitativo, baseado na modelagem estatística de dados como, por exemplo, Bengtsson [1] investigou padrões e propostas de padronização relacionadas ao RCM e descreveu vários aspectos organizacionais considerados ao decidir implementar o RCM. Jardine et al. [2] revisaram a pesquisa sobre diagnósticos e prognósticos de sistemas mecânicos implementando 
a manutenção centrada na confiabilidade com ênfase em modelos, algoritmos e tecnologias para processamento de dados e tomada de decisões de manutenção . Kothamasu et al. [3] revisaram as filosofias e técnicas de monitoramento e prognóstico de todo o sistema. Eles pesquisaram o monitoramento de paradigmas e examinou os detalhes das ferramentas de monitoramento por completo. Além disso, eles introduziram estudos de caso anteriores ao monitoramento e controle do sistema . Também vemos que, Grobal et al. [4] introduziu a arquitetura inicial do framework CBM (Comitê Brasileiro de Metrologia), que estava sendo realizada em um projeto conjunto com a pesquisa SAP, sendo este um sistema integrado de gestão empresarial. Eles mencionaram vários aspectos do CBM: identificação de indicadores, medição de indicadores, modelagem de indicadores, previsão de indicadores e tomada de decisão.

Dragomir et al. [5] analisaram e discutiram o processo prognóstico ou seja uma previsão baseada em fatos e dados reais e atuais, que pode indicar o provável estágio futuro de um processo sob diferentes pontos de vista: o conceito, as medidas e as ferramentas. Eles definiram um quadro para realizar (e desenvolver) sistemas de prognóstico real, e também descreveram o conceito de 'prognóstico' em detalhes e fizeram uma análise das ferramentas usadas no prognóstico e na previsão.

A Aliança de Sistemas Abertos de Gerenciamento de Informações de Máquinas (MIMOSA) propôs e facilitou convenções, diretrizes e recomendações que promovem a unificação econômica de informações de máquinas, avaliação de condições e tecnologia de controle [6]. Recentemente, Hashemian e feijão[7] classificaram as técnicas de Manutenção Centrada na Confiabilidade em três categorias com base em sua fonte de dados: (1) a técnica de manutenção baseada em sensores existente; (2) a técnica de manutenção baseada no sensor de teste; e (3) a técnica de manutenção baseada no sinal de teste.

Prajapati et al. [8] forneceram uma breve visão geral das plantas da CBM. Dieulle et al. [9] propuseram um modelo matemático para determinar uma política de CBM de forma eficiente usando a teoria dos processos de renovação. Em seu modelo, eles consideraram o limiar de substituição preventiva e o cronograma de inspeção como variáveis de decisão. Koç e Lee [10] abordaram o conceito de manutenção preventiva via web em um sistema inteligente de manutenção eletrônica que é implementado via Internet e mostrou seus elementos do sistema .

Além disso, Hirable et al. [11] descreveram o esquema e os requisitos para o agente de peças que faz uma recomendação sobre a manutenção da peça com base nas informações coletadas da Internet e dos dados históricos. Eles mencionaram que o agente de peças poderia calcular o custo de reposição com base na deterioração das peças sobressalentes e suas taxas de reposição. Deuteranopic et al. [12] propuseram a estrutura do agente watchdog para a manutenção preditiva baseada em condições, realizando a avaliação multisensor e a previsão do desempenho da máquina ou do processo. O conceito de agente watchdog baseou sua avaliação de degradação nas leituras de múltiplos sensores que mede as propriedades críticas do processo ou maquinário sob um ambiente de rede e sem barreiras.

O agente watchdog é um sistema embarcado que possui algoritmos para avaliar e prever de forma autônoma a degradação do desempenho e a vida útil restante de máquinas e componentes. Yan et al. [13] apresentaram um método prognóstico para detecção de degradação de máquina, que pode avaliar o desempenho da máquina e prever a vida útil remanescente. Em seu modelo, o desempenho em tempo reavaliado pela inserção de recursos de dados on-line no modelo logístico. E a vida restante é estimada usando um modelo ARMA baseado no histórico de desempenho da máquina. Além disso, $\mathrm{Fu}$ et al. [14] propuseram uma estrutura de manutenção preditiva para a unidade geradora hidrelétrica. Eles apresentaram três elementos-chave para a manutenção preditiva, como monitoramento e previsão, diagnóstico e prognóstico e tomada de decisão. Alias, Bansal et al. [15] descreveram um sistema de manutenção preditiva em tempo real para sistemas de máquinas . 
O objetivo do sistema proposto é localizar e detectar condições elétricas anormais, a fim de prever anormalidades mecânicas que indiquem, ou possam levar à falha de um equipamento . Eles usaram uma abordagem de rede neural para prever parâmetros de uma máquina. Recentemente, Lee et al. [16] introduziu o campo emergente de e-manutenção e seus elementos críticos . Eles também introduziram ferramentas de avaliação e previsão de desempenho, como redes neurais, lógica difusa, regressão logística, modelos de marcas ocultas e redes de crenças bayesianas para avaliação e previsão contínuas do desempenho de um determinado produto .

\section{1 . Padrões internacionais relacionados}

Existem vários padrões internacionais relacionados à abordagem da Confiabilidade. A Tabela 1 mostra os padrões internacionais. Alguns são os padrões de monitoramento e diagnóstico de condições para a indústria de maquinários, por exemplo, ISO 13372, ISO 13373, ISO 13380 e ISO 13381. Em particular, o ISO TC 108 lida com vibrações mecânicas e choques. Como resultado, a ISO 13374 aborda o MIMOSA OSA-CBM representando formatos e métodos para comunicação, apresentação e exibição de informações e dados relevantes. Existem também padrões relacionados às questões de integração e compartilhamento de dados entre instalações de fabricação, por exemplo, ISO 18435 (MIMOSA OSA-EAI). Recentemente, não apenas a indústria de maquinário, mas também a indústria de engenharia de plantas, por exemplo, a indústria de petróleo, petroquímica e gás natural , começam a ter mais interesse na manutenção centrada na confiabilidade devida sua importância e melhora na disponibilidade, como você pode ver na ISO 14224.

Tabela 1. Levantamento de padrões internacionais.

\begin{tabular}{|c|c|}
\hline Padrões & Sujeito \\
\hline $\begin{array}{l}\text { IEEE } \\
1451\end{array}$ & Interface de transdutor inteligente para sensores e atuadores \\
\hline $\begin{array}{l}\text { IEEE } \\
1232\end{array}$ & $\begin{array}{l}\text { Intercâmbio de Inteligência Artificial e Atendimento a Todos os } \\
\text { Ambientes de Teste }\end{array}$ \\
\hline $\begin{array}{l}\text { ISO } \\
13372\end{array}$ & $\begin{array}{l}\text { Monitoramento de condições e diagnóstico de máquinas - } \\
\text { Vocabulário }\end{array}$ \\
\hline $\begin{array}{l}\text { ISO } \\
13373-1\end{array}$ & $\begin{array}{l}\text { Monitoramento de condições e diagnóstico de máquinas - } \\
\text { Monitoramento de condições de vibração - Parte } 1 . \\
\text { Procedimentos gerais }\end{array}$ \\
\hline $\begin{array}{l}\text { ISO } \\
13373-2\end{array}$ & $\begin{array}{l}\text { Monitoramento de condições e diagnóstico de máquinas - } \\
\text { Monitoramento de condições de vibração - Parte } 2 . \\
\text { Processamento, análise e apresentação de dados de vibração }\end{array}$ \\
\hline $\begin{array}{l}\text { ISO } \\
13374\end{array}$ & $\begin{array}{l}\text { Formatos e métodos MIMOSA OSA-CBM para comunicação, } \\
\text { apresentação e exibição de informações e dados relevantes }\end{array}$ \\
\hline $\begin{array}{l}\text { ISO } \\
13380\end{array}$ & $\begin{array}{l}\text { Monitoramento de condição e diagnóstico de máquinas - } \\
\text { diretrizes gerais sobre o uso de parâmetros de desempenho }\end{array}$ \\
\hline $\begin{array}{l}\text { ISO } \\
13381-1\end{array}$ & $\begin{array}{l}\text { Monitoramento e diagnóstico de condições de máquinas - } \\
\text { Prognóstico, diretrizes gerais }\end{array}$ \\
\hline $\begin{array}{l}\text { ISO } \\
14224\end{array}$ & $\begin{array}{l}\text { Indústrias de petróleo, petroquímica e gás natural - coleta e troca } \\
\text { de dados de confiabilidade e manutenção para equipamentos }\end{array}$ \\
\hline
\end{tabular}




\begin{tabular}{|l|l|}
\hline Padrões & Sujeito \\
\hline $\begin{array}{l}\text { ISO } \\
17359\end{array}$ & $\begin{array}{l}\text { Monitoramento de condições e diagnóstico de máquinas - } \\
\text { diretrizes gerais }\end{array}$ \\
\hline $\begin{array}{l}\text { ISO } \\
18435\end{array}$ & $\begin{array}{l}\text { Integração de aplicativos de diagnóstico e manutenção MIMOSA } \\
\text { OSA-EAI }\end{array}$ \\
\hline ISO & Gestão de ativos \\
\hline
\end{tabular}

\subsection{Técnicas para Confiabilidade}

Existem vários tipos de técnicas a serem aplicadas no processamento de dados, diagnósticos e prognósticos para a implementação do CBM, conforme mostrado na Tabela. No CBM, existem três tipos de abordagem: (1) abordagem baseada em dados, (2) abordagem baseada em modelos e (3) abordagem híbrida [17]. De acordo com Caesarendra [18], a abordagem baseada em dados tem a capacidade de transformar dados de alta dimensão em informações de menor dimensão. É também conhecida como a abordagem de mineração de dados ou a abordagem de aprendizado de máquina, que usa dados históricos para aprender automaticamente um modelo de comportamento do sistema [19] . No entanto, esta abordagem tem a dependência da qualidade dos dados operacionais e há na compreensão física do produto designado. Pelo contrário, a abordagem baseada em modelo tem a capacidade de incorporar a compreensão física do produto alvo. Ele se baseia no uso de um modelo analítico (conjunto de equações algébricas ou diferenciais) para representar o comportamento do sistema, incluindo o fenômeno da degradação [20]. Mas, tem a limitação no ponto em que só pode ser aplicado a tipos específicos de produtos. A Tabela 3 mostra várias técnicas para cada abordagem.

Tabela 2. Levantamento de técnicas de manutenção baseadas em condições.

\begin{tabular}{|c|c|}
\hline Estágio & Técnicas \\
\hline Processamento de dados & $\begin{array}{l}\text { Filtragem de Kalman } \\
\text { - } \\
\text { Momentos de tempo-frequência / tempo- } \\
\text { frequência } \\
\text { - } \\
\text { Análise wavelet } \\
\text { - } \\
\text { Modelo autorregressivo (AR) } \\
\text { - } \\
\text { Análise de Fourier } \\
\text { - } \\
\text { Análise de Wigner-Ville }\end{array}$ \\
\hline Diagnóstico & $\begin{array}{l}\text { Lógica difusa } \\
\text { - } \\
\text { Rede neural artificial } \\
\text { - } \\
\text { Algorítmos genéticos } \\
\text { - } \\
\text { Reconhecimento estatístico de padrões }\end{array}$ \\
\hline
\end{tabular}




\begin{tabular}{|l|l|}
\hline Estágio & Técnicas \\
\hline \multirow{4}{*}{ Prognósticos } & - \\
& Modelo de Markov escondido \\
& - \\
& Máquina de vetores de suporte \\
& - \\
& Indução da árvore de decisão \\
& - \\
& Regressão logística \\
\hline & - \\
& Rede neural artificial \\
& - \\
& Teoria da confiabilidade \\
& - \\
& Análise estatística (por exemplo, regressão) \\
& - \\
& Análise de dados de séries temporais \\
& - \\
& Raciocínio Baseado em Casos (CBR) \\
\hline \multirow{2}{*}{ Operação } & - \\
& Teoria da Renovação \\
& - \\
& Programação matemática \\
& - \\
& Simulação \\
& - \\
& Tomada de decisão multicritério (MCDM) \\
&
\end{tabular}

Tabela 3. Levantamento de técnicas de manutenção baseadas em condições.

\begin{tabular}{|c|c|}
\hline Classificação & Técnicas \\
\hline $\begin{array}{l}\text { Abordagem orientada } \\
\text { por modelo }\end{array}$ & $\begin{array}{l}\text { Baseado em física } \\
\text { Técnicas clássicas de IA (sistemas especialistas } \\
\text { baseados em regras, máquinas de estados finitos, } \\
\text { raciocínio qualitativo) [21] }\end{array}$ \\
\hline $\begin{array}{l}\text { Abordagem orientada } \\
\text { por dados }\end{array}$ & $\begin{array}{l}\text { Algoritmos numéricos convencionais (regressão } \\
\text { linear, filtros de Kalman) [21] } \\
\text { Abordagem estatística (método estatístico } \\
\text { multivariado, modelos de espaço de estado, modelo } \\
\text { regressivo) [22] } \\
\text { Aprendizado de máquina (redes neurais, árvores de } \\
\text { decisão, máquinas de vetores de suporte) [21] } \\
\text { Rede bayesiana baseada em RNA, modelo oculto de } \\
\text { Markov, análise de componentes principais, modelo } \\
\text { Gray [22] }\end{array}$ \\
\hline $\begin{array}{l}\text { Abordagem Baseada } \\
\text { no Conhecimento }\end{array}$ & $\begin{array}{l}\text { Sistemas especialistas [22] } \\
\text { Lógica difusa [22] }\end{array}$ \\
\hline
\end{tabular}




\section{MATERIAIS E MÉTODOS}

A aplicação da metodologia FMEA juntamente com os estudos de caso da CBM permitiu diagnosticar as causas que estavam gerando a irregularidade no acabamento final da peça (Cilindros). Como apoio no desenvolvimento a esta metodologia FMEA e fortalecimento do diagnóstico, aplicouse técnicas de manutenção preditiva (análise de vibração) com periodicidade trimestral monitorando todos os conjuntos e subconjuntos do equipamento, permitindo identificar a evolução dos parâmetros de vibração em RMS e aceleração $(\mathrm{Ge})$ do equipamento com valores globais subindo de forma exponencial colocando a nível crítico de falha. A Figura 2 mostra a Tabela FMEA aplicada para identificar modo de efeito e causa.

\begin{tabular}{|c|c|c|c|c|c|c|c|c|c|c|c|c|c|c|c|}
\hline \multicolumn{16}{|c|}{ FMEA } \\
\hline \multicolumn{5}{|c|}{ Núm. do FMEA: 001} & \multicolumn{5}{|c|}{ Preparado por: Fabio Assisi, 7393} & & \multicolumn{5}{|c|}{ Datad de revisäo do FMEA: Ver OO } \\
\hline \multicolumn{5}{|c|}{ Identificaçăa do item: Torno CNC DH 1450} & \multicolumn{5}{|c|}{ Datade conclusäo:09/11/2011 } & & \multicolumn{5}{|l|}{ Pág, 01 de 01.} \\
\hline \multicolumn{5}{|c|}{ Modelo / Produto: Rolamento INA81244 P5 } & \multicolumn{5}{|c|}{ Dtado FMEA: 20/11/2011 } & & & & & & \\
\hline \multicolumn{5}{|c|}{ Departamento responsável: Forjaria e Usinagens } & \multicolumn{5}{|c|}{ Equipe:'Manutençäo USP } & & & & & & \\
\hline \multirow{2}{*}{$\begin{array}{l}\text { Funģoode } \\
\text { projeto }\end{array}$} & \multirow{2}{*}{$\begin{array}{c}\text { Modo } \\
\text { Potencial } \\
\text { de Falha }\end{array}$} & \multirow{2}{*}{\begin{tabular}{|l|} 
Efétito \\
Potencial \\
defallha
\end{tabular}} & \multirow{2}{*}{$\Delta$} & & \multirow{2}{*}{\begin{tabular}{|c|} 
Causa \\
Potencial de \\
Fallha
\end{tabular}} & \multirow{2}{*}{ Ocorrência } & \multirow{2}{*}{$\begin{array}{c}\text { Metodo } \\
\text { de } \\
\text { detecção }\end{array}$} & \multirow{2}{*}{ RPN } & \multirow{2}{*}{ Açä Recomen. } & \multirow{2}{*}{$\begin{array}{l}\text { Resp. e Oatade } \\
\text { condusiono }\end{array}$} & \multicolumn{5}{|c|}{ Resultado da Aç̄o. } \\
\hline & & & & & & & & & & & Açâo Tomadas & Severidade & Ocorrência & Deteçää & RPN \\
\hline \multirow[t]{2}{*}{ Carga axia } & $\begin{array}{l}\text { 1- Faltade } \\
\text { lubrificaçäla }\end{array}$ & Rigosidade & s & 8 & Sobrecarga & 1 & 2 & 16 & 1-Rever proc. Op & $\begin{array}{l}\text { 1. Eng, Process } \\
20 / 01 / 2012\end{array}$ & \begin{tabular}{|l|} 
1- Revisiso do \\
procediment
\end{tabular} & 4 & 1 & 2 & 8 \\
\hline & $\begin{array}{c}2 \cdot \text { Carga } \\
\text { axial } \\
\text { excessiva }\end{array}$ & & & & & & & & 2.Subs: Rolamento & $\begin{array}{l}\text { 2-MNT,Cil } \\
\text { 28/01/2012 }\end{array}$ & $\begin{array}{l}2 \text {-Subsituir } \\
\text { rolamento. }\end{array}$ & 6 & 2 & 1 & 12 \\
\hline
\end{tabular}

Figura 2. FMEA aplicada para identificar modo de efeito e causa

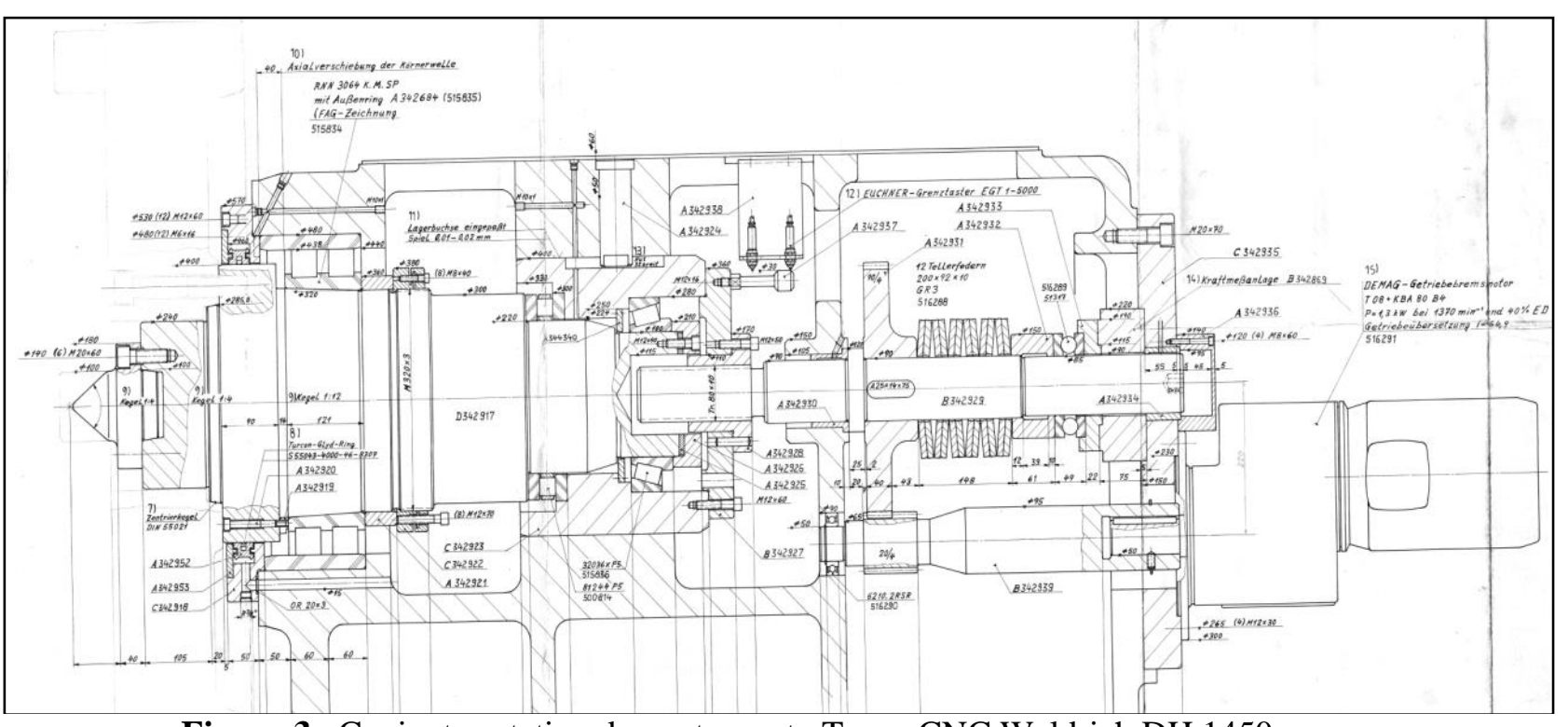

Figura 3. Conjunto rotativo do contraponto Torno CNC Waldrich DH 1450 


\section{ISSN $2447-5378$}

\section{RESULTADOS E DISCUSSÕES}

Neste item, são mostrados os resultados obtidos através da atuação da Engenharia de Preditiva onde identificou junto ao projeto da máquina os conjunto e subconjunto sujeitos a falhas durante o processo de usinagem, conforme mostra a Figura 3. Nos Gráficos 1 e 2 foi possível identificar uma elevada tendência de falha que ultrapassou do limite de alarme 1 para nível de alarme 2, num período de monitoração de seis meses. Após as coletas nos respectivos períodos e análises em FFT (Espectro), identificou-se uma aceleração de falha em um dos rolamentos monitorados (INA 81244 P5) do tipo axial de dupla carreira de rolos, conforme mostra gráfico 3 a seguir, localizada na manga do contraponto do torno CNC Waldrich conforme figura 3, a Figura 4 mostra o estado do rolamento danificado e os Gráficos 4 e 5 mostras condição de partida da máquina após a intervenção.
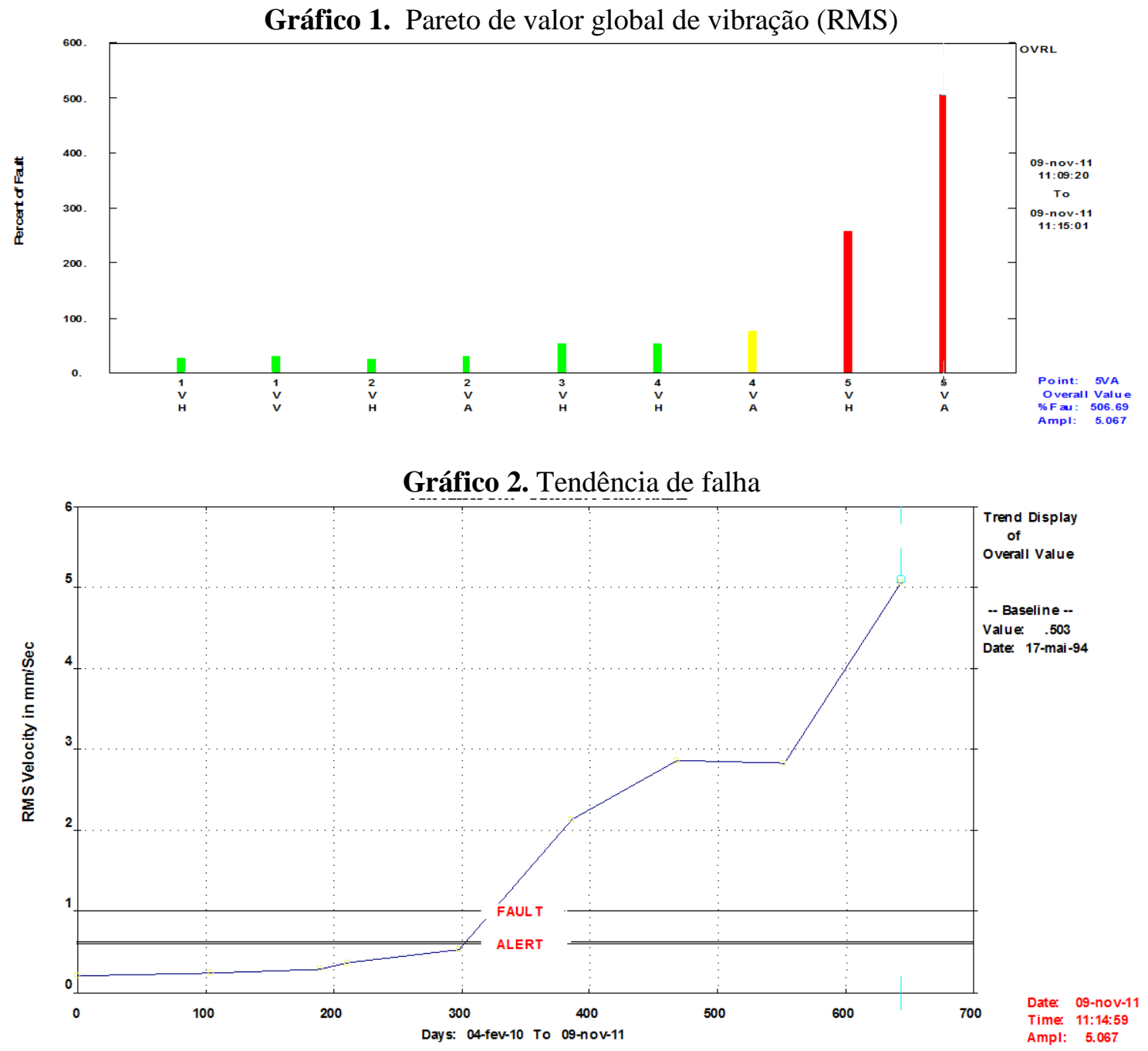
Gráfico 3. Curva Espectral

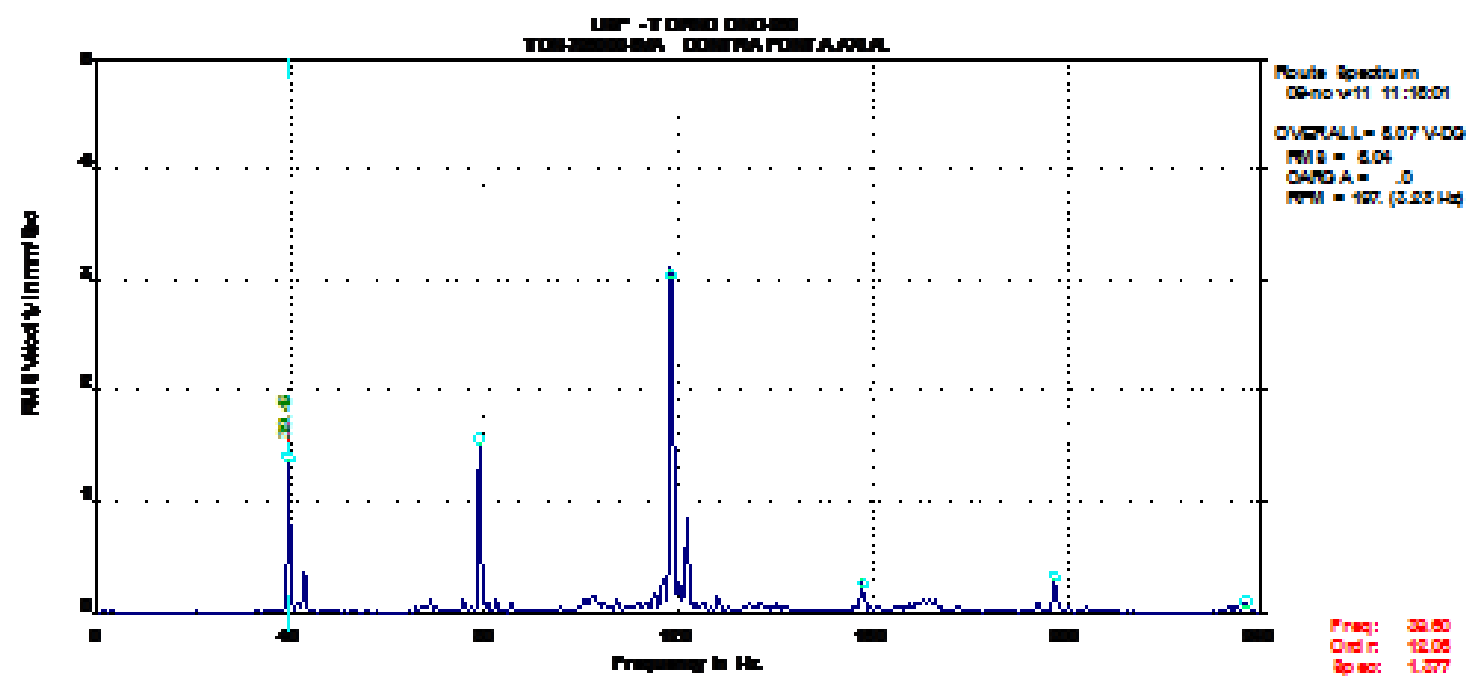

Gráfico 4. Queda na ocorrência de falha

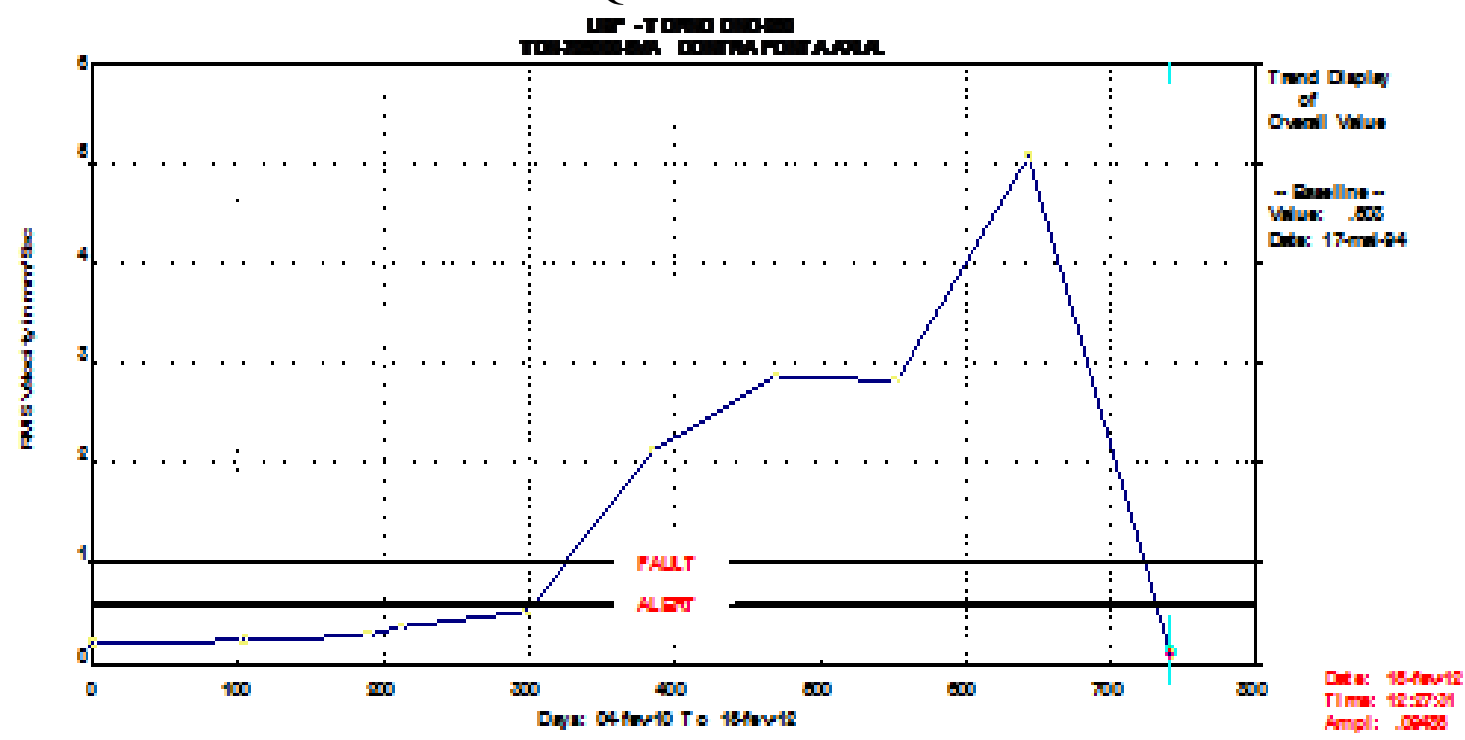

Gráfico 5. Valor global de vibração

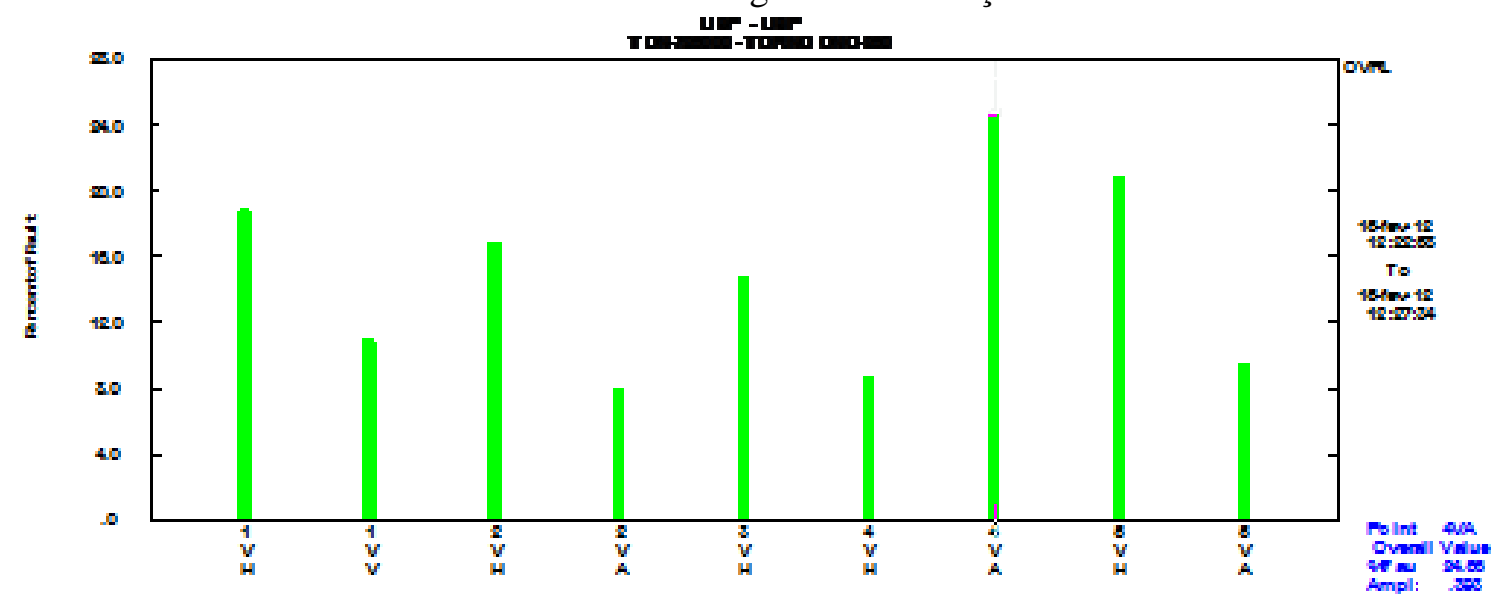




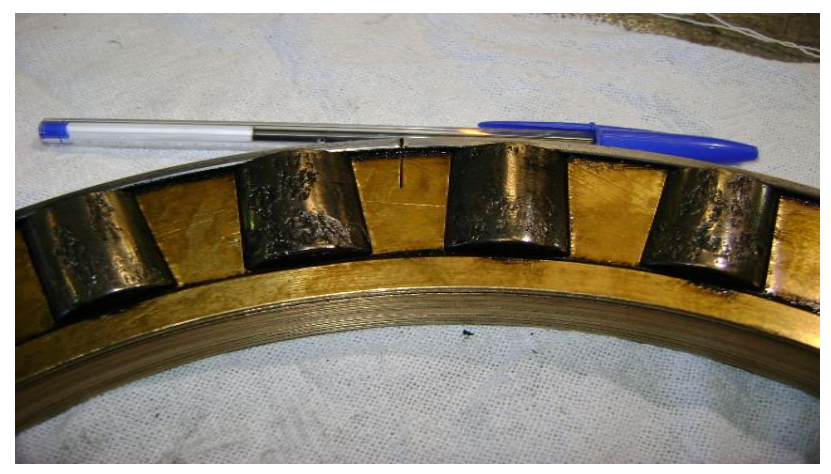

Figura 5. Roletes danificados pego no FFT de aceleração

\section{CONCLUSÃO}

Analisar o modo que uma determinada falha surge e evolui, bem como antecipar quais seriam as consequências para o equipamento se entrasse em colapso devido a tal falha. Verifica-se a importância da análise de modo e efeito na prevenção de novas ocorrências semelhantes. A partir da análise de vibrações e tipo construtivo do equipamento em questão pode - se identificar seus possíveis causadores.

É também de grande importância analisar os componentes substituídos / reparados para avaliação das características do seu modo de falha e possível causa.

Conclui-se que com este estudo a importância de se utilizar uma metodologia eficaz que norteia a utilizar a melhor pratica de manutenção, assim como, um bom programa para soluções de problemas. Com a aplicação do FMEA diagnosticamos as causas de falhas do equipamento durante a usinagem, nos possibilitando aplicar a Técnica de Análise de Vibração para identificar a origem da falha apresentada, com auxílio da Engenharia de Preditiva foi possível realizar um diagnóstico conclusivo da falha. Foi proposto após o presente estudo a troca do rolamento identificado. Desta forma fica evidenciada a eficácia de ferramentas e metodologias utilizadas que direcionaram os pontos passivos de melhoria.

\section{REFERÊNCIAS}

[1] D. Bansal, DJ Evans, B. Jones Um sistema de manutenção preditiva em tempo real para sistemas de máquinas. Revista Internacional de Máquinas-Ferramenta e Fabricação , 44 ( 2004 ), pp. $759-766$

[2] H. Hiraoka , N. Iwanami , Y. Fujii , T. Seya , H. IshizukaAgentes de rede para suporte de ciclo de vida de peças mecânicas. In: Anais do Terceiro Simpósio Internacional sobre Design Ambientalmente Consciente e Manufatura Inversa ( 2003 ), pp. 61 - 64

[3] M. Koc, J. LeeUma estrutura de sistema para sistemas de manutenção eletrônica de próxima geração. Transação do engenheiro mecânico chinês, 12 ( 2001 )

[4] C. Fu, L. Ye, Y. Liu, R. Yu, B. Iung, Y. Cheng, et al.Manutenção preditiva em sistema inteligente de gerenciamento de manutenção de controle para unidade geradora de energia hidroelétrica. IEEE Transactions on Conversão de Energia , 19 ( 1 ) ( 2004 ), pp. 179 - 186

[5] Dragomir OE, Gouriveau R, F Dragomir, Minca E, Zerhouni N. Revisão do problema prognóstico na manutenção baseada na condição. In: Procedimentos da Conferência Europeia de Controlo; 2009; Budapeste, Hungria. 


\section{ISSN $2447-5378$}

[6] R. Kothamasu, SH Huang, WH Verduin Monitoramento e prognóstico da saúde do sistema uma revisão dos atuais paradigmas e práticas. Revista Internacional de Tecnologia Avançada de Manufatura, 28 ( 2006 ), pp. 1012 - 1024

[7] HM Hashemian, WC BeanTécnicas de manutenção preditiva de última geração. IEEE Transactions on Instrumentation and Measurement, 60 ( 10 ) ( 2011 ), pp. 3480 - 3492

[8] A. Prajapati , J. Bechtel , S. GanesanManutenção baseada na condição: uma pesquisa. Revista de Qualidade em Engenharia de Manutenção , 18 ( 4 ) ( 2012 ), pp. 384 - 400

[9] Dieulle L, B’erenguer, C, Gral A, Roussignol M. Programação contínua de manutenção preditiva de tempo para um sistema em deterioração. In: Anais do Simpósio Anual do IEEE sobre Confiabilidade e Sustentabilidade; 2001; p. 150-155. [10] M. Koc, J. LeeUma estrutura de sistema para sistemas de manutenção eletrônica de próxima geração. Transação do engenheiro mecânico chinês , 12 ( 2001 )

[11] H. Hiraoka, N. Iwanami , Y. Fujii , T. Seya, H. Ishizuka Agentes de rede para suporte de ciclo de vida de peças mecânicas. In: Anais do Terceiro Simpósio Internacional sobre Design Ambientalmente Consciente e Manufatura Inversa ( 2003 ), pp. 61 - 64

[12] D. Djurdjanovic, J. Lee,Agente de Vigilância deJ. Ni- uma abordagem de prognóstico baseada em infotrônica para avaliação e previsão da degradação do desempenho do produto Avançado Engenharia Informática , 17 ( 2003 ), pp. 109 - 125

[13] J. Yan, M. Koç, J. Lee Algoritmo de prognóstico para avaliação de desempenho de máquinas e sua aplicação. Planejamento e Controle de Produção , 15 ( 8 ) ( 2004 ), pp. 796 - 801

[14] C. Fu , L. Ye, Y. Liu , R. Yu , B. Iung, Y. Cheng, et al. Manutenção preditiva em sistema inteligente de gerenciamento de manutenção de controle para unidade geradora de energia hidroelétrica. IEEE Transactions on Conversão de Energia , 19 ( 1 ) ( 2004 ), pp. 179 - 186

[15] D. Bansal, DJ Evans , B. Jones Um sistema de manutenção preditiva em tempo real para sistemas de máquinas. Revista Internacional de Máquinas-Ferramenta e Fabricação , 44 ( 2004 ), pp. 759 - 766

[16] J. Lee, J. Ni , D. Djurdjanovic , H. Qiu , Ferramentas inteligentes do prognóstico de H. Abd Liao e e-manutenção. Computadores na Indústria , 57 ( 2006 ), pp. 476 - 489

[17] J. LeeTeleservice engenharia na manufatura: desafios e oportunidades. Revista Internacional de Máquinas-Ferramenta \& Fabricação , 38 ( 8 ) ( 1998 ), pp. 901 - 910

[18] Abordagembaseada em modelos e baseada em dados deW. Caesarendrapara prognósticos de máquina. (Dissertação de Mestrado). Universidade Nacional de Pukyoung ( 2010 )

[19] Schwabacher MA. Uma pesquisa de prognósticos baseados em dados. In: Anais da Conferência AIAA Infotech @ Aerospace; Virginia, EUA; 2005.

[20] D. Tobon-Mejia , Medjaher K. , N. Zerhouni , G. TripotUm método de prognóstico de falha orientada a dados com base na mistura de gaussianos ocultos modelos de Markov. IEEE Transactions on Reliability , 61 ( 2 ) ( 2012 ), pp. 491 - 503

[21] Poongodai A, Reader SB. Técnica AI em diagnósticos e prognósticos. In: Anais da $2^{\mathrm{a}}$ Conferência Nacional sobre Computação Futura; 2013.

[22] Y. Peng, M. Dong, MJ ZuoStatus atual dos prognósticos da máquina na manutenção baseada em condições: uma revisão.Revista Internacional de Tecnologia de Fabricação Avançada, 50 ( 2010 ), pp. 297 - 313 